OPEN ACCESS

Edited by:

Ping-Chih Ho,

University of Lausanne, Switzerland

Reviewed by:

Laura Solt,

Scripps Research Institute, USA

Chia-Lin Hsu,

National Yang-Ming University,

Taiwan

Guoliang Cui,

Deutsches Krebsforschungszentrum

(DKFZ), Germany

*Correspondence:

Markus Kleinewietfeld markus.kleinewietfeld@vib-

uhasselt.be

Specialty section:

This article was submitted to Molecular Innate Immunity,

a section of the journal

Frontiers in Immunology

Received: 06 January 2017 Accepted: 06 March 2017

Published: 21 March 2017

Citation:

Binger KJ, Côrte-Real BF and

Kleinewietfeld M (2017)

Immunometabolic Regulation of

Interleukin-17-Producing T Helper

Cells: Uncoupling New Targets for

Autoimmunity.

Front. Immunol. 8:311.

doi: 10.3389/fimmu.2017.00311

\section{Immunometabolic Regulation of Interleukin-17-Producing T Helper Cells: Uncoupling New Targets for Autoimmunity}

\author{
Katrina J. Binger ${ }^{1}$, Beatriz F. Côrte-Real² and Markus Kleinewietfeld ${ }^{2 *}$ \\ ${ }^{1}$ Department of Biochemistry and Molecular Biology, Bio21 Institute of Molecular Science and Biotechnology, University of \\ Melbourne, Parkville, VIC, Australia, ${ }^{2}$ VIB Laboratory of Translational Immunomodulation, Hasselt University, BIOMED, \\ Diepenbeek, Belgium
}

Interleukin-17-producing T helper (Th17) cells are critical for the host defense of bacterial and fungal pathogens and also play a major role in driving pathogenic autoimmune responses. Recent studies have indicated that the generation of Th17 cells from naiive $\mathrm{CD}^{+} \mathrm{T}$ cells is coupled with massive cellular metabolic adaptations, necessary to cope with different energy and metabolite requirements associated with switching from a resting to proliferative state. Furthermore, Th17 cells have to secure these metabolic adaptations when facing nutrient-limiting environments, such as at the sites of inflammation. Accumulating data indicates that this metabolic reprogramming is significantly linked to the differentiation of T helper cells and, particularly, that the metabolic changes of Th17 cells and anti-inflammatory Forkhead box $\mathrm{P}^{+}{ }^{+}$regulatory $\mathrm{T}$ cells are tightly and reciprocally regulated. Thus, a better understanding of these processes could offer potential new targets for therapeutic interventions for autoimmune diseases. In this mini-review, we will highlight some of the recent advances and discoveries in the field, with a particular focus on metabolic demands of Th17 cells and their implications for autoimmunity.

Keywords: glycolysis, oxidative phosphorylation, immunometabolism, interleukin-17-producing $\mathrm{T}$ helper cells, regulatory $\mathrm{T}$ cells, autoimmune diseases

\section{INTRODUCTION}

T cells respond to alterations in the host environment via multiple steps of activation, proliferation, and finally differentiation into specialized subsets, which are exquisitely programmed to deal with the challenge at hand; whether it is the presence of an intracellular or extracellular pathogen, or other alterations in tissue homeostasis, which may require either inflammatory or regulatory responses. These various responses are tightly regulated by the interplay of specialized $\mathrm{CD} 4^{+}$effector T helper (Th) cell subsets and antiinflammatory Forkhead box $\mathrm{P}^{+}\left(\mathrm{FOXP}^{+}\right)$regulatory T cells (Tregs) (1).

One such type of a specialized $\mathrm{T}$ effector cell subset consists of interleukin (IL)-17-producing $\mathrm{T}$ helper (Th17) cells. In mice, Th17 cells could be differentiated from stimulated naive $\mathrm{CD}^{+} \mathrm{T}$ cells in the presence of the pleiotropic cytokine transforming growth factor (TGF)- $\beta 1$ in combination with IL-6. Th17 cells are specialized to respond against certain bacterial and fungal pathogens in the tissue sites in which they are located, namely the mucosal linings of the gut and airway epithelia 
(2). However, Th17 cells are also known for their pathogenic potential against the host, due to their association with several autoimmune diseases such as multiple sclerosis (MS), psoriasis, and rheumatoid arthritis (RA) (1-4). More recent studies have demonstrated that Th17 cells can be heterogeneous in phenotype and function and can even show antiinflammatory properties. This pro-inflammatory versus antiinflammatory/ homeostatic phenotype of Th17 cells seems to be determined by a set of specific signaling modules, where pathogenicity is critically influenced by a high level of expression of IL-23 receptor, granulocyte-macrophage colony-stimulating factor, and Th1-like transcripts [e.g., interferon- $\gamma$, T-box transcription factor 21 (TBX21/Tbet)] and by the absence of the antiinflammatory cytokine IL-10 (5). The induction of these different Th17 phenotypes can be mimicked in vitro by varying the combination of stimulatory triggers and cytokines. For example, the stimulation of naive $\mathrm{T}$ cells with a combination of IL-1 $\beta$, IL- 6 , and IL-23 in the absence of TGF- $\beta 1$ induces the differentiation of Th17 cells that exhibit a highly pro-inflammatory and pathogenic phenotype, compared to "classically" TGF- $\beta 1$ + IL- 6 differentiated Th17 cells (6-8). Together, these studies illustrate that the pro-inflammatory potential of Th17 cells is extremely sensitive to the presence and combinations of stimulatory cues within the local microenvironment.

Of course in vivo, the tissue site in which Th17 cells are generated does not only consist of cytokines but is also packed with a variety of small molecules, including metabolites. This "metabolomic" milieu is dynamically changing in both its composition and concentration, which may have various effects on Th17 cells, particularly through their cellular metabolism (9-12). This is highlighted by many recent studies showing even further variation in Th17 phenotype can been fashioned in vitro by the addition of e.g., fatty acids $(13,14)$, phospholipids (15), cholesterol intermediates $(16)$, oxysterols $(17,18)$, and even electrolytes such as sodium or potassium $(19,20)$. In this mini-review, we will highlight some recent advances in understanding the metabolic adaptations and mechanisms employed by $\mathrm{T}$ cells when they undergo activation and differentiate into specialized subsets, focusing on Th17 cells.

\section{METABOLIC ADAPTATIONS OF Th CELLS}

The overall aim of cellular metabolism, independently of the cell type, is to generate energy [adenosine triphosphate (ATP)] and metabolites, which are essential for cells to perform various functions, sustain life and growth. Glucose is the major cellular fuel source, and it is broken down into ATP by two separate, but connected pathways: glycolysis and oxidative phosphorylation (OXPHOS). In glycolysis, glucose is broken via 10 enzymatic steps down to pyruvate, yielding two ATP molecules; a process that does not require oxygen. Most cells go on to oxidize pyruvate in the tricarboxylic acid cycle, subsequently fueling mitochondrial OXPHOS, which, in an oxygen-dependent process, yields more than 30 ATP. Alternatively, pyruvate can be converted to lactate, which ultimately feeds back into glycolysis. While glycolysis produces far less ATP, it has several advantages in that it is fast and generates metabolites, so under oxygen-poor conditions, it can suffice to provide energy and necessary biomolecules for the cell $(11,12,21)$.

A metabolic phenomena first observed in cancerous cells by Otto Warburg in 1924 is when proliferating cells preferentially utilize glycolytic metabolism even when oxygen is abundant (22). This aerobic glycolysis, often called "Warburg" metabolism, while bioenergetically less efficient, results in an additional benefit from increased flux into new molecules (nucleotides, amino acids, and fatty acids), as well as pathways involved in generation of redox-protective metabolites. It is now apparent that the activation of $\mathrm{T}$ cells induces this same metabolic switch to aerobic glycolysis (Figure 1). In fact, this was first observed more than 50 years ago that the activation of $\mathrm{T}$ cells, such as with mitogens like concanavalin A, results in immense changes in glycolytic cellular metabolism (23-27). More recently, Frauwirth et al. demonstrated the importance of costimulation in initiating these metabolic adaptations (28). Costimulation of CD3 and CD28 induced increased expression of the glucose transporter 1 (Glut1), subsequently leading to augmented glucose uptake and flux through glycolysis. The same has been reported for the expression of amino acid transporters and amino acid uptake (29, 30). Functionally, this provides the activated T cell with ATP, but more importantly, the accumulation of nutrients and biomass permits the activated $\mathrm{T}$ cell to expand and rapidly proliferate. Adopting an aerobic glycolytic metabolism is now appreciated to be a feature of all Th cell populations $(9-11,21)$. Furthermore, this switch to aerobic glycolysis is not only limited to proliferating immune cells, as the activation of myeloid cells like dendritic cells (31) and macrophages (32) with toll-like receptor agonists also induces these same metabolic adaptations, indicating that this mechanism may infer a general requirement for cells of the immune system.

\section{Th17 CELLS VERSUS TREG: A CHOICE IN CELLULAR METABOLISM}

For differentiation into special $\mathrm{T}$ effector cell subsets, cellular metabolism remains a process that is distinct and necessary for the acquisition of downstream specialized functions. This is none the more apparent when comparing the metabolic configurations of Th17 cells and Tregs. As a consequence of costimulation in combination with TGF- $\beta$ and IL-6, the transcription factor RARrelated orphan receptor gamma $\mathrm{t}(\mathrm{ROR} \gamma \mathrm{t})$ is activated leading to the generation of Th17 cells. Conversely, while maintaining a requirement for TGF- $\beta$ in the absence of other cytokines, with the exception of IL-2, induced Tregs (iTregs) are generated via the activation of the transcription factor FOXP3. Metabolically, these two subsets are incredibly distinct $(13,33,34)$. In vitro stimulation of naïve $\mathrm{T}$ cells with Th17-stimulating conditions induces robust glucose uptake and a shift to aerobic glycolysis. By contrast, Tregs instead increase their lipid uptake and utilize energy-efficient pathways such as fatty acid oxidation (FAO) and mitochondrial OXPHOS (Figure 1) (10-12, 21).

The key factors essential for the regulation of Treg and Th17 cell metabolic reprogramming have now been identified as a network of metabolic kinases that function as nutrient/energy sensors and metabolic transcription factors, in line with their regulation of 


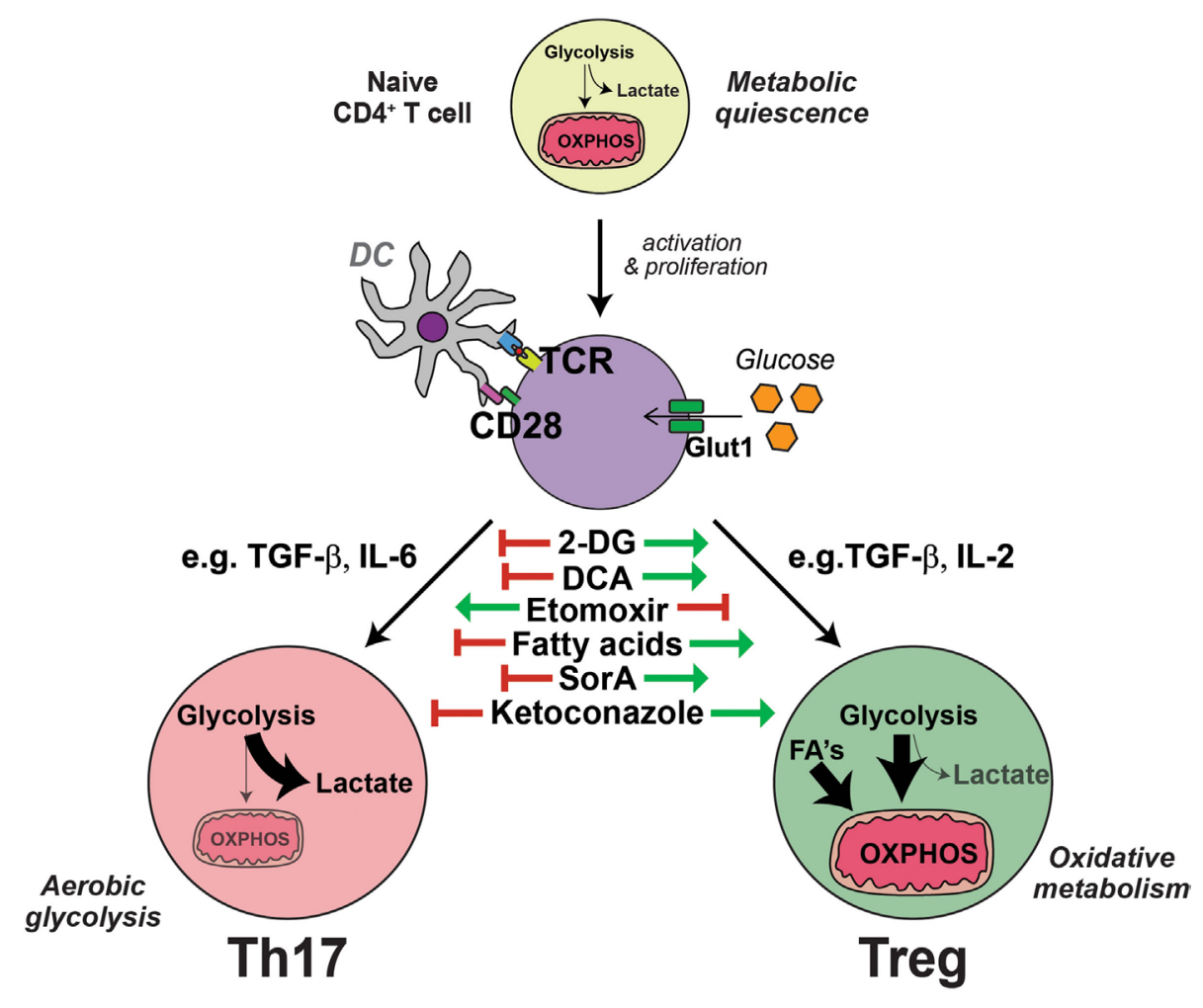

Pathogenic

Regulatory

FIGURE 1 | Interleukin-17-producing T helper (Th17) cells and regulatory T cells (Tregs) have distinct metabolic requirements. The presentation of antigen and costimulation by dendritic cells induce the reprogramming of T cells into an aerobic glycolytic "Warburg" metabolism, characterized by the increased expression of glucose transporter 1 (Glut1) and augmented glucose uptake. Generally, in the presence of transforming growth factor (TGF)- $\beta$ with the combination of other cytokines such as interleukin (IL)-6, activated T cells are polarized into specialized Th17 cells, which have an even further enhanced glycolytic metabolism and flux into intermediate pathways, required for the generation of amino acids, nucleotides, and fatty acids essential for Th17 effector function. Conversely, the induction of Tregs from activated T cells by TGF- $\beta$ and the combination of other cytokines, such as IL-2, results in an increased uptake of fatty acids (FA) and an elevated lipid and mitochondrial oxidative metabolism. Within each subset, there exists further heterogeneity, depending on the presence of further local stimulatory cues, such as other cytokines and small metabolites, thus generating pathogenic or non-pathogenic Th17 cells and various polarized regulatory Treg cells; all of which exhibit further metabolic complexity. Th17 cells are dependent on aerobic glycolytic metabolism, as inhibiting glycolysis with 2-deoxyglucose (2-DG) or dichloroacetate (DCA) prevents Th17 cell generation even in the presence of Th17 cell-promoting conditions. Likewise, the addition of exogenous fatty acids or soraphen A (SorA), which enhances lipid oxidative metabolism, similarly inhibits Th17 cell generation. These metabolic interventions have the opposite effect on the generation of Tregs, which are conversely enhanced by treatments that augment lipid oxidative metabolism and blunted by inhibitors of lipid transport such as etomoxir. Cholesterol derivatives are essential for Th17 cell differentiation and blockade of cholesterol biosynthesis, for example, with ketoconazole, inhibits the generation of Th17 cells but has no effect on Tregs.

nutrient transporter expression (9-12, 21, 35). Major regulatory checkpoints are mechanistic/mammalian target of rapamycin (mTOR), the kinase complex that is a promoter of aerobic glycolysis and anabolic metabolism after stimulation of T cells and the AMP-activated protein kinase (AMPK) complex that promotes FAO and catabolic metabolism [reviewed in Ref. (9-12, 21, 35)]. Particularly for Th17 cells, the metabolic transcription factor, hypoxia-inducible factor 1 (HIF- $1 \alpha$ ), seems to play a special role (36). Under Th17-promoting conditions, HIF- $1 \alpha$ expression is rapidly increased in an mTOR-dependent manner, and its deletion prevents the generation of Th17 cells both in vitro and in Th17-promoting disease models in vivo $(34,37)$. As HIF- $1 \alpha$ is a transcription factor regulating the expression of metabolic enzymes (36), Shi et al. hypothesized its importance in regulating the cellular metabolic reprogramming of Th17 cells (34). Deletion of HIF-1 $\alpha$ under Th17-promoting conditions results in a blunted upregulation of Glut1 and the reduced expression of crucial glycolytic enzymes such as hexokinase 2, phosphofructokinase 1 , and lactate dehydrogenase. Together, these data indicate that indeed HIF- $1 \alpha$ is an essential facilitator of the acquisition of Th17 glycolytic metabolism (34). In line with this, a recent study identified another important regulatory checkpoint for Th17 cell or Treg-specific metabolic pathway decisions. Gerriets et al. identified pyruvate dehydrogenase $(\mathrm{PDH})$ and the pyruvate metabolism as a key decisive point between $\mathrm{T}$ cell glycolytic and oxidative metabolism. The conversion of cytosolic pyruvate into mitochondrial acetyl-CoA is catalyzed by $\mathrm{PDH}$ for oxidative metabolism and is inhibited by $\mathrm{PDH}$ kinase (PDHK). PDHK is 
regulated by hypoxia and HIF-1 $\alpha$ and promotes the generation of lactate by suppressing pyruvate oxidation (38). By a detailed metabolic analysis, they identified with PDHK1 an isoform that is predominantly expressed in Th17 cells but not in Th1 cells or Tregs. The inhibition of PDHK1 by dichloroacetate (DCA) was able to suppress glycolysis and selectively affected the generation and survival of Th17 cells in part through the generation of reactive oxygen species (ROS) (38).

By contrast, both in vitro generated iTregs and ex vivo isolated thymic-derived Tregs exhibit increased phosphorylation of AMPK (13), a broad sensor of decreased cellular nutrients and energy (39). Subsequently, treatment in vivo with an AMPK agonist, metformin, resulted in an increased generation of Tregs (13). However, conversely, AMPK-knockout CD4 ${ }^{+}$ $\mathrm{T}$ cells did not demonstrate deficient Treg generation (40). An explanation for these discrepant results may be because AMPK has a much broader role in $\mathrm{T}$ cell metabolic adaptations, not only within the Treg subset. Recently, Blagih et al. showed that $\mathrm{T}$ cells deficient in AMPK are unable to adapt in vitro changes in the availability of nutrients such as glucose and glutamine. Subsequently, this translated to an impaired ability of both Th1 and Th17 generation in vivo (41), pointing toward multiple layers of cellular metabolic sensing and adaptation in the acquisition of $\mathrm{T}$ effector function, particularly for Th17 cells. In line with this, another metabolic checkpoint for fate decisions between Tregs and Th17 cells was recently described (33). De novo fatty acid synthesis (FAS), which is inhibited by AMPK (42), was shown to be essential for the generation of Th17 cells in contrast to Tregs (33). Accordingly, the inhibition or deletion of acetyl-CoA carboxylase 1 (ACC1), a key enzyme for de novo FAS, resulted in an impaired Th17 differentiation, whereas Tregs were induced $(21,33,43)$.

These findings highlight how the generation of Tregs and Th17 cells is tightly linked to their metabolic state, offering potential new targets for the regulation of these two reciprocally regulated $\mathrm{T}$ cell subsets (Figure 1). Indeed, it was shown already in addition to the targeting of AMPK with metformin that the inhibition of mTOR by rapamycin could block Th17 cell generation while favoring Tregs (11). Moreover, the inhibition of lipid oxidation with drugs such as etomoxir, which inhibits the activity of carnitine palmitoyltransferase I, resulting in a reduction of the fatty acid import into the mitochondria (44), impairs the differentiation of iTregs but has no effect on the generation of Th17 cells $(13,44)$. Conversely, inhibiting glucose metabolism with 2-deoxyglucose impairs Th17 cell differentiation while reciprocally promoting iTreg induction (34). Similarly, the addition of exogenous fatty acids (13) or the inhibition of de novo FAS with small-molecule metabolic modulators like soraphen A (SorA) (33) impairs Th17 cell differentiation and promotes Tregs, even in the presence of pro-Th17-inducing conditions. This does not mean, however, that Th17 cells do not require lipids for their metabolic remodeling. It has been recently shown that oxysterols, such as 27-dihydroxycholesterol (18), metabolites in the cholesterol biosynthetic pathway (16), and desmosterol (17) bind to, and agonize ROR $\gamma \mathrm{t}$ activity, subsequently promoting the differentiation of Th17 cells. Thus, inhibiting cholesterol synthesis, for example with ketoconazole, impairs Th17 differentiation and IL-17 production, but has no effect on Treg differentiation (17). Moreover, PDHK1 blockade by DCA was able to selectively impair Th17 cell function and ameliorated experimental autoimmune encephalomyelitis (EAE) without having significant effects on Tregs or Th1 cells (38).

\section{Th17 METABOLISM: A POTENTIAL THERAPEUTIC TARGET FOR THE TREATMENT OF AUTOIMMUNE DISEASES?}

Under homeostatic conditions, Th17 cells are easily able to undergo the critical metabolic changes required for their specialized function described above, as nutrients are plentiful. However, under altered conditions (e.g., infection, disease, diet), metabolic restrictions may occur, which could have significant bearings on Th17 cell function. These recent discoveries discussed before highlight the intimate connectivity between immunity and cellular metabolism leading to the following questions: (a) Do disturbances in $\mathrm{T}$ cell metabolism contribute to the development of human autoimmune diseases? (b) Can Th17 metabolism be therapeutically targeted, thereby manipulating the immune system to become less inflammatory, and thus providing protection or treatment for autoimmune diseases with strong Th17 components such as RA and MS?

Besides in animal models of disease, it has additionally been demonstrated that several autoimmune diseases go in line with metabolic alterations and that also $\mathrm{T}$ cells of patients have dysregulated metabolic profiles, indicating that indeed an altered T cell metabolism is associated with disease. This was for instance already shown for RA, systemic lupus erythematosus, and MS $(11,12,45-47)$. Albeit for most of the diseases premature and incompletely understood, the notion of altered metabolic profiles in human autoimmunity points towards intriguing new avenues for therapeutic interventions. In fact, recent studies have generated promising experimental data indicating the feasibility of this hypothesis. Most of the current reports concentrated here on the reciprocal regulation in regards of cellular metabolism between Tregs and Th17 cells, since Th17 cells primarily depend on glycolysis and Tregs seem to utilize mainly FAO. As described above, the key targets here would be the inhibition of the mTOR/ HIF- $1 \alpha$ pathway to prevent the development of the initiation of a "pro-inflammatory" metabolic signature or the activation of AMPK to influence cellular metabolism favoring the generation of antiinflammatory Tregs. In line with this, the interference of de novo FAS by inhibiting ACC1 with the inhibitor SorA was demonstrated to be effective at promoting the generation of Tregs, as well as inhibiting Th17 cells (33). However, the major problem, as for many other currently available immunotherapies, remains specificity. The above-mentioned metabolic reactions are all important in other $\mathrm{T}$ cell subsets and immune cells, and therefore, unspecific targeting of these pathways bears the risk of general immunosuppression and/or the interference with the generation of memory cells, which seem to have similar metabolic demands as Tregs; not to mention effects on non-immune cells $(9,10,12,21)$. Moreover, it remains to be seen on how other 
Th cell subsets react and in addition, importantly, on how these pathways interfere with pathogenic and regulatory/homeostatic subsets of Th17 cells (5). In this respect, it is of interest that it has already been demonstrated that an altered lipid biosynthesis and the fatty acid and cholesterol composition of the cell could impact Th17 cell pathogenicity by generating endogenous ROR $\gamma \mathrm{t}$ ligands (15-18).

It is also important to note that for many of the studies described in this review, there are only sparse data on the contribution of these same metabolic pathways to the differentiation of human Tregs and Th17 cells, and the contribution of these molecules to disease in vivo is not clear. Recent studies have, for instance, shown that in contrast to findings in murine Tregs, human Tregs in fact highly depend on glycolysis $(46,48)$. Similarly, murine Tregs have also been shown to be glycolytic in vivo (49). This obvious discrepancy of human to murine Tregs might be associated with the existence of different FOXP3 splice variants in humans, which could dictate their function in contrast to mice $(50,51)$. It was shown that glycolysis through the glycolytic enzyme enolase-1 controls the induction of the FOXP3 splice variant containing exon 2 that is crucial for human Treg function (46). Therefore, defining metabolic targets depending only on in vitro findings and experimental animal models must be carefully examined if they can be fully translated to the setting of human disease. Promising experimental strategies to cope with these problems might be the inclusion of different mouse models expressing different FOXP3 splice variants or the use of humanized mouse models, in combination with the analysis of patient samples as recently demonstrated by Yang et al. for RA (52). This elegant study identified that patient samples exhibited a dysregulation in the pentose phosphate pathway (PPP), which was associated with a depletion in ROS. Insufficient oxidative signaling resulted in ataxia telangiectasia mutated kinase-dependent bypass of the $\mathrm{G}_{2} / \mathrm{M}$ cell cycle, consequently leading to hyperproliferation and a shift of RA patients into a highly pathogenic Th17/Th1 cell profile. Importantly, this dysregulation could be rescued by metabolic interventions that restored the ROS pool: supplementation with menadione (vitamin K3), disrupting synthesis of the ROS quencher glutathione, or by blocking glucose shunting into the PPP, which was even demonstrated in a humanized mouse model in vivo, transplanted with synovial tissue and T cells of RA patients $(47,52)$.

\section{CONCLUSION}

The research on immunometabolism and its relation to disease is an extremely fast growing and promising field, which may offer novel therapeutic avenues for immunomodulation in settings of various human diseases. Particularly in autoimmune diseases like RA or MS, metabolic disturbances influencing the Treg/Th17 axis may play a role, and we are just at the beginning to understand the tight linkage between metabolic pathways and immune cell function.

\section{REFERENCES}

1. Kleinewietfeld M, Hafler DA. The plasticity of human Treg and Th17 cells and its role in autoimmunity. Semin Immunol (2013) 25(4):305-12. doi:10.1016/j.smim.2013.10.009
While this increased knowledge of the metabolic requirements and alterations of Th17 cells and Tregs in autoimmunity provides many specific targets for intervention, it may also offer indirect dietary approaches that beneficially influence dysregulated $\mathrm{T}$ cell metabolism by systemically changing nutrient or metabolite availability. This is not a new concept, as it is well known that nutrition and the general metabolic condition of individuals has a profound impact on the immune system. Malnutrition, particularly prominent in developing countries, is clearly associated with reduced immune function. By contrast, obesity, which is associated with a "Western" lifestyle and a nutritional pattern high in calories, fat, and salt, goes in hand with the onset of a low-grade systemic inflammation $(12,53,54)$. While obesity is well known to predispose individuals to diabetes and cardiovascular diseases, it additionally seems to represent a risk factor for inflammatory autoimmune diseases like MS $(53,55,56)$. This may be driven largely by the strong effect of obesity on the Treg/ Th17 axis through various metabolites or adipokines like leptin and through pro-inflammatory cytokines like IL-6 $(53,57)$. An additional molecular mechanism contributing to this effect was recently described by Endo et al. Here, mice fed a high-fat diet had an elevated induction of Th17 differentiation, in a mechanism that was reliant on the expression of ACC1, which subsequently contributed to ROR $\gamma$ t activation (43). Therefore, it is important to understand the direct and indirect influences of diet on the immune system and Treg/Th17 axis, or more broadly, the connection of systemic and cellular metabolism and its relation to human autoimmune disease (45). A variety of studies have indicated that particularly dietary interventions could have severe effects on autoimmunity by influencing metabolic parameters of immune cells. It is thus tempting to speculate that, for instance, dietary interventions like fasting mimicking diets, that were recently shown to be beneficial in EAE and MS patients by shifting the Treg/Th17 balance (58), or by lowering the sodium content in diets, that had a strong metabolic impact on macrophages (59, 60 ), could serve as interesting alternatives or additions for drugs directly targeting cellular metabolism.

\section{AUTHOR CONTRIBUTIONS}

All authors listed have made substantial, direct, and intellectual contribution to the work and approved it for publication.

\section{FUNDING}

KJB was supported by a National Health and Medical Research Council of Australia Early Career Fellowship (APP1037633). MK was supported by the European Research Council (ERC) under the European Union's Horizon 2020 research and innovation program (640116), by a SALK-grant from the government of Flanders, Belgium and by an Odysseus-grant of the Research Foundation Flanders, Belgium (FWO).

2. Korn T, Bettelli E, Oukka M, Kuchroo VK. IL-17 and Th17 cells. Annu Rev Immunol (2009) 27:485-517. doi:10.1146/annurev.immunol.021908.132710

3. Binger KJ, Linker RA, Muller DN, Kleinewietfeld M. Sodium chloride, SGK1, and Th17 activation. Pflugers Arch (2015) 467(3):543-50. doi:10.1007/ s00424-014-1659-z 
4. Luchtman DW, Ellwardt E, Larochelle C, Zipp F. IL-17 and related cytokines involved in the pathology and immunotherapy of multiple sclerosis: current and future developments. Cytokine Growth Factor Rev (2014) 25(4):403-13. doi:10.1016/j.cytogfr.2014.07.013

5. Burkett PR, Meyer zu Horste G, Kuchroo VK. Pouring fuel on the fire: Th17 cells, the environment, and autoimmunity. JClin Invest (2015) 125(6):2211-9. doi:10.1172/JCI78085

6. Chung Y, Chang SH, Martinez GJ, Yang XO, Nurieva R, Kang HS, et al. Critical regulation of early Th17 cell differentiation by interleukin-1 signaling. Immunity (2009) 30(4):576-87. doi:10.1016/j.immuni.2009.02.007

7. Ghoreschi K, Laurence A, Yang XP, Tato CM, McGeachy MJ, Konkel JE, et al. Generation of pathogenic $\mathrm{T}(\mathrm{H}) 17$ cells in the absence of TGF-beta signalling. Nature (2010) 467(7318):967-71. doi:10.1038/nature09447

8. Lee Y, Awasthi A, Yosef N, Quintana FJ, Xiao S, Peters A, et al. Induction and molecular signature of pathogenic TH17 cells. Nat Immunol (2012) 13(10):991-9. doi:10.1038/ni.2416

9. Pearce EL, Pearce EJ. Metabolic pathways in immune cell activation and quiescence. Immunity (2013) 38(4):633-43. doi:10.1016/j.immuni.2013.04.005

10. Park BV, Pan F. Metabolic regulation of T cell differentiation and function. Mol Immunol (2015) 68(2 Pt C):497-506. doi:10.1016/j.molimm.2015.07.027

11. Wang R, Solt LA. Metabolism of murine TH 17 cells: impact on cell fate and function. Eur J Immunol (2016) 46(4):807-16. doi:10.1002/eji.201545788

12. MacIver NJ, Michalek RD, Rathmell JC. Metabolic regulation of T lymphocytes. Annu Rev Immunol (2013) 31:259-83. doi:10.1146/ annurev-immunol-032712-095956

13. Michalek RD, Gerriets VA, Jacobs SR, Macintyre AN, MacIver NJ, Mason EF, et al. Cutting edge: distinct glycolytic and lipid oxidative metabolic programs are essential for effector and regulatory CD4+ T cell subsets. J Immunol (2011) 186(6):3299-303. doi:10.4049/jimmunol.1003613

14. Haghikia A, Jorg S, Duscha A, Berg J, Manzel A, Waschbisch A, et al. Dietary fatty acids directly impact central nervous system autoimmunity via the small intestine. Immunity (2015) 43(4):817-29. doi:10.1016/j.immuni.2015.09.007

15. Wang C, Yosef N, Gaublomme J, Wu C, Lee Y, Clish CB, et al. CD5L/AIM regulates lipid biosynthesis and restrains Th17 cell pathogenicity. Cell (2015) 163(6):1413-27. doi:10.1016/j.cell.2015.10.068

16. Santori FR, Huang P, van de Pavert SA, Douglass EF Jr, Leaver DJ, Haubrich $\mathrm{BA}$, et al. Identification of natural RORgamma ligands that regulate the development of lymphoid cells. Cell Metab (2015) 21(2):286-97. doi:10.1016/j. cmet.2015.01.004

17. Hu X, Wang Y, Hao LY, Liu X, Lesch CA, Sanchez BM, et al. Sterol metabolism controls $\mathrm{T}(\mathrm{H}) 17$ differentiation by generating endogenous RORgamma agonists. Nat Chem Biol (2015) 11(2):141-7. doi:10.1038/nchembio.1714

18. Soroosh P, Wu J, Xue X, Song J, Sutton SW, Sablad M, et al. Oxysterols are agonist ligands of RORgammat and drive Th17 cell differentiation. Proc Natl Acad Sci U S A (2014) 111(33):12163-8. doi:10.1073/pnas.1322807111

19. Kleinewietfeld M, Manzel A, Titze J, Kvakan H, Yosef N, Linker RA, et al. Sodium chloride drives autoimmune disease by the induction of pathogenic TH17 cells. Nature (2013) 496(7446):518-22. doi:10.1038/nature1 1868

20. Eil R, Vodnala SK, Clever D, Klebanoff CA, Sukumar M, Pan JH, et al. Ionic immune suppression within the tumour microenvironment limits $\mathrm{T}$ cell effector function. Nature (2016) 537(7621):539-43. doi:10.1038/nature19364

21. Almeida L, Lochner M, Berod L, Sparwasser T. Metabolic pathways in T cell activation and lineage differentiation. Semin Immunol (2016) 28(5):514-24. doi:10.1016/j.smim.2016.10.009

22. Vander Heiden MG, Cantley LC, Thompson CB. Understanding the Warburg effect: the metabolic requirements of cell proliferation. Science (2009) 324(5930):1029-33. doi:10.1126/science.1160809

23. Cooper EH, Barkhan P, Hale AJ. Observations on the proliferation of human leucocytes cultured with phytohaemagglutinin. Br J Haematol (1963) 9:101-11. doi:10.1111/j.1365-2141.1963.tb05446.x

24. Culvenor JG, Weidemann MJ. Phytohaemagglutinin stimulation of rat thymus lymphocytes glycolysis. Biochim Biophys Acta (1976) 437(2):354-63. doi:10.1016/0304-4165(76)90005-2

25. Hedeskov CJ. Early effects of phytohaemagglutinin on glucose metabolism of normal human lymphocytes. Biochem J (1968) 110(2):373-80. doi:10.1042/ bj1100373

26. Roos D, Loos JA. Changes in the carbohydrate metabolism of mitogenically stimulated human peripheral lymphocytes. I. Stimulation by phytohaemagglutinin. Biochim Biophys Acta (1970) 222(3):565-82. doi:10.1016/0304-4165(70)90182-0

27. Sagone AL Jr, LoBuglio AF, Balcerzak SP. Alterations in hexose monophosphate shunt during lymphoblastic transformation. Cell Immunol (1974) 14(3):443-52. doi:10.1016/0008-8749(74)90195-6

28. Frauwirth KA, Riley JL, Harris MH, Parry RV, Rathmell JC, Plas DR, et al. The CD28 signaling pathway regulates glucose metabolism. Immunity (2002) 16(6):769-77. doi:10.1016/S1074-7613(02)00323-0

29. Carr EL, Kelman A, Wu GS, Gopaul R, Senkevitch E, Aghvanyan A, et al. Glutamine uptake and metabolism are coordinately regulated by ERK/ MAPK during T lymphocyte activation. J Immunol (2010) 185(2):1037-44. doi:10.4049/jimmunol.0903586

30. Sinclair LV, Rolf J, Emslie E, Shi YB, Taylor PM, Cantrell DA. Control of amino-acid transport by antigen receptors coordinates the metabolic reprogramming essential for T cell differentiation. Nat Immunol (2013) 14(5):500-8. doi:10.1038/ni.2556

31. Krawczyk CM, Holowka T, Sun J, Blagih J, Amiel E, DeBerardinis RJ, et al. Tolllike receptor-induced changes in glycolytic metabolism regulate dendritic cell activation. Blood (2010) 115(23):4742-9. doi:10.1182/blood-2009-10-249540

32. Rodriguez-Prados JC, Traves PG, Cuenca J, Rico D, Aragones J, Martin-Sanz P, et al. Substrate fate in activated macrophages: a comparison between innate, classic, and alternative activation. J Immunol (2010) 185(1):605-14. doi:10.4049/jimmunol.0901698

33. Berod L, Friedrich C, Nandan A, Freitag J, Hagemann S, Harmrolfs K, et al. De novo fatty acid synthesis controls the fate between regulatory $\mathrm{T}$ and $\mathrm{T}$ helper 17 cells. Nat Med (2014) 20(11):1327-33. doi:10.1038/nm.3704

34. Shi LZ, Wang R, Huang G, Vogel P, Neale G, Green DR, et al. HIFlalphadependent glycolytic pathway orchestrates a metabolic checkpoint for the differentiation of TH17 and Treg cells. JExp Med (2011) 208(7):1367-76. doi:10.1084/jem.20110278

35. Yang $\mathrm{K}$, Chi H. mTOR and metabolic pathways in $\mathrm{T}$ cell quiescence and functional activation. Semin Immunol (2012) 24(6):421-8. doi:10.1016/j. smim.2012.12.004

36. Semenza GL. Regulation of mammalian $\mathrm{O}_{2}$ homeostasis by hypoxia-inducible factor 1. Annu Rev Cell Dev Biol (1999) 15:551-78. doi:10.1146/annurev. cellbio.15.1.551

37. Dang EV, Barbi J, Yang HY, Jinasena D, Yu H, Zheng Y, et al. Control of $\mathrm{T}(\mathrm{H}) 17 / \mathrm{T}(\mathrm{reg})$ balance by hypoxia-inducible factor 1. Cell (2011) 146(5):77284. doi:10.1016/j.cell.2011.07.033

38. Gerriets VA, Kishton RJ, Nichols AG, Macintyre AN, Inoue M, Ilkayeva O, et al. Metabolic programming and PDHK1 control CD4+ T cell subsets and inflammation. J Clin Invest (2015) 125(1):194-207. doi:10.1172/JCI76012

39. Hardie DG, Ross FA, Hawley SA. AMPK: a nutrient and energy sensor that maintains energy homeostasis. Nat Rev Mol Cell Biol (2012) 13(4):251-62. doi:10.1038/nrm 3311

40. MacIver NJ, Blagih J, Saucillo DC, Tonelli L, Griss T, Rathmell JC, et al. The liver kinase $\mathrm{B} 1$ is a central regulator of $\mathrm{T}$ cell development, activation, and metabolism. J Immunol (2011) 187(8):4187-98. doi:10.4049/jimmunol.1100367

41. Blagih J, Coulombe F, Vincent EE, Dupuy F, Galicia-Vazquez G, Yurchenko $\mathrm{E}$, et al. The energy sensor AMPK regulates $\mathrm{T}$ cell metabolic adaptation and effector responses in vivo. Immunity (2015) 42(1):41-54. doi:10.1016/j. immuni.2014.12.030

42. Yuan HX, Xiong Y, Guan KL. Nutrient sensing, metabolism, and cell growth control. Mol Cell (2013) 49(3):379-87. doi:10.1016/j.molcel.2013.01.019

43. Endo Y, Asou HK, Matsugae N, Hirahara K, Shinoda K, Tumes DJ, et al. Obesity drives Th17 cell differentiation by inducing the lipid metabolic kinase, ACC1. Cell Rep (2015) 12(6):1042-55. doi:10.1016/j.celrep.2015.07.014

44. Xu FY, Taylor WA, Hurd JA, Hatch GM. Etomoxir mediates differential metabolic channeling of fatty acid and glycerol precursors into cardiolipin in H9c2 cells. JLipid Res (2003) 44(2):415-23. doi:10.1194/jlr.M200335JLR200

45. Norata GD, Caligiuri G, Chavakis T, Matarese G, Netea MG, Nicoletti A, et al. The cellular and molecular basis of translational immunometabolism. Immunity (2015) 43(3):421-34. doi:10.1016/j.immuni.2015.08.023

46. De Rosa V, Galgani M, Porcellini A, Colamatteo A, Santopaolo M, Zuchegna $\mathrm{C}$, et al. Glycolysis controls the induction of human regulatory $\mathrm{T}$ cells by modulating the expression of FOXP3 exon 2 splicing variants. Nat Immunol (2015) 16(11):1174-84. doi:10.1038/ni.3269 
47. Tsokos GC. Metabolic control of arthritis: switch pathways to treat. Sci Transl Med (2016) 8(331):331fs8. doi:10.1126/scitranslmed.aaf4953

48. Procaccini C, Carbone F, Di Silvestre D, Brambilla F, De Rosa V, Galgani M, et al. The proteomic landscape of human ex vivo regulatory and conventional T cells reveals specific metabolic requirements. Immunity (2016) 44(2):406-21. doi:10.1016/j.immuni.2016.01.028

49. Gerriets VA, Kishton RJ, Johnson MO, Cohen S, Siska PJ, Nichols AG, et al. Foxp3 and toll-like receptor signaling balance Treg cell anabolic metabolism for suppression. Nat Immunol (2016) 17(12):1459-66. doi:10.1038/ni.3577

50. Smith EL, Finney HM, Nesbitt AM, Ramsdell F, Robinson MK. Splice variants of human FOXP3 are functional inhibitors of human CD4+ T-cell activation. Immunology (2006) 119(2):203-11. doi:10.1111/j.1365-2567.2006.02425.x

51. Walker MR, Kasprowicz DJ, Gersuk VH, Benard A, Van Landeghen M, Buckner JH, et al. Induction of FoxP3 and acquisition of T regulatory activity by stimulated human CD4+CD25- T cells. J Clin Invest (2003) 112(9):1437-43. doi:10.1172/JCI19441

52. Yang Z, Shen Y, Oishi H, Matteson EL, Tian L, Goronzy JJ, et al. Restoring oxidant signaling suppresses proarthritogenic $\mathrm{T}$ cell effector functions in rheumatoid arthritis. Sci Transl Med (2016) 8(331):331ra38. doi:10.1126/ scitranslmed.aad7151

53. Manzel A, Muller DN, Hafler DA, Erdman SE, Linker RA, Kleinewietfeld M. Role of "Western diet" in inflammatory autoimmune diseases. Curr Allergy Asthma Rep (2014) 14(1):404. doi:10.1007/s11882-013-0404-6

54. Gerriets VA, MacIver NJ. Role of T cells in malnutrition and obesity. Front Immunol (2014) 5:379. doi:10.3389/fimmu.2014.00379

55. Mokry LE, Ross S, Timpson NJ, Sawcer S, Davey Smith G, Richards JB. Obesity and multiple sclerosis: a Mendelian Randomization Study. PLoS Med (2016) 13(6):e1002053. doi:10.1371/journal.pmed.1002053
56. Sumarac-Dumanovic M, Stevanovic D, Ljubic A, Jorga J, Simic $M$, Stamenkovic-Pejkovic D, et al. Increased activity of interleukin-23/interleukin-17 proinflammatory axis in obese women. Int J Obes (Lond) (2009) 33(1):151-6. doi:10.1038/ijo.2008.216

57. Winer S, Paltser G, Chan Y, Tsui H, Engleman E, Winer D, et al. Obesity predisposes to Th17 bias. Eur J Immunol (2009) 39(9):2629-35. doi:10.1002/ eji.200838893

58. Choi IY, Piccio L, Childress P, Bollman B, Ghosh A, Brandhorst S, et al. A diet mimicking fasting promotes regeneration and reduces autoimmunity and multiple sclerosis symptoms. Cell Rep (2016) 15(10):2136-46. doi:10.1016/j. celrep.2016.05.009

59. Binger KJ, Gebhardt M, Heinig M, Rintisch C, Schroeder A, Neuhofer W, et al. High salt reduces the activation of IL-4- and IL-13-stimulated macrophages. J Clin Invest (2015) 125(11):4223-38. doi:10.1172/JCI80919

60. Jorg S, Grohme DA, Erzler M, Binsfeld M, Haghikia A, Muller DN, et al. Environmental factors in autoimmune diseases and their role in multiple sclerosis. Cell Mol Life Sci (2016) 73(24):4611-22. doi:10.1007/s00018-016-2311-1

Conflict of Interest Statement: The authors declare that the research was conducted in the absence of any commercial or financial relationships that could be construed as a potential conflict of interest.

Copyright (C) 2017 Binger, Côrte-Real and Kleinewietfeld. This is an open-access article distributed under the terms of the Creative Commons Attribution License (CC $B Y)$. The use, distribution or reproduction in other forums is permitted, provided the original author(s) or licensor are credited and that the original publication in this journal is cited, in accordance with accepted academic practice. No use, distribution or reproduction is permitted which does not comply with these terms. 